\title{
Infinite-Dilution Viscoelastic Properties of Comb-Shaped Polystyrenes
}

\author{
Yutaka Mitsuda, John L. Schrag, and John D. Ferry \\ Department of Chemistry and Rheology Research Center, \\ University of Wisconsin, Madison, Wisconsin 53706, U.S.A.
}

(Received March 5, 1973)

\begin{abstract}
The storage and loss shear moduli, $G^{\prime}$ and $G^{\prime \prime}$, have been measured for solutions of three comb-shaped polystyrenes with numbers of branches $(f) 8.3,24$, and 51 , by use of the Birnboim-Schrag multiple-lumped resonator. The weight-average molecular weights were $0.51,1.38$, and $6.64 \times 10^{6}$; the fractions of backbone mass were $0.11,0.04$, and 0.014 respectively. The frequency range was 100 to $6000 \mathrm{~Hz}$ and the concentration range 0.0016 to $0.013 \mathrm{~g} / \mathrm{m} l$. Decalin was used for one sample $(f=51)$ as a $\Theta$-solvent at $15^{\circ} \mathrm{C}$; toluene, $\alpha$-chloronaphthalene, and Aroclor 1232 were used as good solvents with different viscosities, the latter at several different temperatures. The extrapolated intrinsic moduli, $\left[G^{\prime}\right]$ and $\left[G^{\prime \prime}\right]$, were compared with the Zimm-Kilb theory as evaluated by Osaki and Schrag for star-shaped and by Osaki for comb-shaped molecules. At low frequencies, $\left[G^{\prime}\right]$ and the reduced steady-state shear compliance $j_{\mathrm{eR}}^{0}$ were not as much smaller than those for a corresponding linear molecule as these theories would predict, and were surprisingly insensitive to the degree of branching. At higher frequencies, the logarithmic slopes of $\left[G^{\prime \prime}\right]$ and $\left[G^{\prime}\right]$ against frequency were unusually large $(0.8)$ and their ratio was also large (about 3). This behavior corresponds to an empirical relaxation spectrum in which the relaxation times $\tau_{p}$ are proportional to $p^{-\alpha}$ with $\alpha=1.25$.

KEY WORDS Viscoelasticity / Dilute Solutions / Multiple-Lumped Resonator / Intrinsic Modulus / Comb-Shaped Polymer / Polystyrene / Zimm-Kilb Theory /
\end{abstract}

Recently, measurements have been made of linear viscoelastic properties of polymer solutions sufficiently dilute to permit extrapolation to infinite dilution for quantitative comparison with molecular theories of polymer dynamics. ${ }^{1}$ Dynamic viscoelastic properties at lower frequencies (i.e., radian frequency $\omega$ less than 10 times the reciprocal of the longest relaxation time) are rather insensitive to details of chemical structure, but depend greatly on molecular topology, so the behavior of branched molecules is of particular interest. Data for star-shaped polybutadiene with 4 branches ${ }^{2}$ and polystyrene with 9 branches ${ }^{3}$ have been reported and compared with the predictions of the Zimm-Kilb theory ${ }^{4}$ as evaluated numerically by Osaki and Schrag; ${ }^{5}$ the agreement was quite good, although for 9 branches the hydrodynamic interaction parameter $h^{*}$ had to be assigned a value somewhat higher than was expected on the basis of previous results on linear polymers. ${ }^{6}$

We now report dilute-solution measurements on three branched polystyrenes with comb struc- tures containing 8,24 , and 51 branches respectively, and compare the results with the ZimmKilb theory.

\section{EXPERIMENTAL}

\section{Materilas}

The comb polystyrenes were generously given us by Professor M. Nagasawa of Nagoya University. They were prepared by anionic polymerization followed by coupling of the branches to the chloromethylated parent (backbone) polymer and subsequent fractionation as described elsewhere. ${ }^{7,8}$ Data for their characterization are summarized in Table I. Here, $\bar{M}_{n, \mathrm{p}}$ and $\bar{M}_{n, \mathrm{~b}}$ are the number-average molecular weights of the parent (backbone) polymers and of the branches coupled to them, respectively, determined osmometrically; ${ }^{7} \bar{M}_{w}$, the weight-average molecular weight of the comb, determined by light scattering; ${ }^{7} f$, the number of branches, $\left(\bar{M}_{w}-\right.$ $\left.\bar{M}_{n, \mathrm{p}}\right) / \bar{M}_{n, \mathrm{~b}} ; \lambda$, the proportion of mass in the backbone, $\bar{M}_{n, \mathrm{p}} / \bar{M}_{w} ;[\eta]$, the intrinsic viscosity 
Table I. Data for characterization of samples

\begin{tabular}{lccc}
\hline \multicolumn{1}{c}{ Sample code } & KI-7 & KI-2 & H-3 \\
\hline $\bar{M}_{n, \mathrm{p}} \times 10^{-4}$ & 5.7 & 5.7 & 9.5 \\
$\bar{M}_{n, \mathrm{~b}} \times 10^{-4}$ & 5.5 & 5.5 & 12.9 \\
$\bar{M}_{w} \times 10^{-4}$ & 51 & 138 & 664 \\
$f$ & 8.3 & 24 & 51 \\
$\lambda$ & 0.11 & 0.041 & 0.014 \\
{$[\eta]$, A1232, $20^{\circ} \mathrm{C}$} & 59 & 101 & 167 \\
{$[\eta], \alpha-\mathrm{CIN}, 25^{\circ} \mathrm{C}$} & & & 193 \\
{$[\eta]$, toluene, $25^{\circ} \mathrm{C}$} & & & 229 \\
{$[\eta]$, Decalin, $15^{\circ} \mathrm{C}$} & & & 69 \\
\hline
\end{tabular}

Table II. Viscosities and densities of solvents

\begin{tabular}{lrll}
\hline \multicolumn{1}{c}{ Solvent } & Temp, ${ }^{\circ} \mathrm{C}$ & $\eta_{\mathrm{s}}$, poise & $\rho, \mathrm{g} / \mathrm{m} l$ \\
\hline$\alpha$-Chloronaphthalene & 25.00 & 0.0302 & 1.195 \\
Toluene & 25.00 & 0.00552 & 0.862 \\
Decalin & 15.00 & 0.0303 & 0.890 \\
Aroclor 1232 & -6.00 & 0.845 & 1.253 \\
& 20.00 & 0.131 & 1.232 \\
& 37.78 & 0.0637 & 1.218 \\
\hline
\end{tabular}

in $\mathrm{m} l / \mathrm{g}$, determined in our laboratory by conventional capillary viscosimetry.

The solvents were used without further purification: $\alpha$-chloronaphthalene, superior grade, Matheson, Coleman, and Bell; toluene, analytical grade, Mallinkrodt; Decalin, approximately half $c i s$ and half trans, practical, Matheson, Coleman, and Bell; Aroclor 1232, lot AK-7, Monsanto. Their viscosities $\left(\eta_{\mathrm{s}}\right)$ and densities $(\rho)$ at the temperatures of measurement are listed in Table II; the former are obtained by the multiple-lumped resonator in the course of the viscoelastic measurements. Decalin is a $\Theta$ solvent for linear polystyrene at $16^{\circ} \mathrm{C}$, and it was estimated that $15^{\circ} \mathrm{C}$ would approximate the $\Theta$-temperature for a branched polymer. ${ }^{9}$ The intrinsic viscosity of sample $\mathrm{H}-3$ in this solvent corresponds to a branched-to-linear ratio $g_{\eta}$ of 0.32 , based on the intrinsic viscosity relation in cyclohexane. ${ }^{8}$

Solutions were made up by weight with very gentle stirring at room temperature, and polymer concentration $(c)$ in $\mathrm{g} / \mathrm{m} l$ was calculated assuming additivity of volumes. The most concentrated solution was measured first and then sequentially diluted to several lower concentrations, usually a total of 5 . The concentration ranges were, for the 8-branch, 0.004 to $0.013 \mathrm{~g} / \mathrm{ml}$; for the 24-branch, 0.0037 to 0.011 ; for the 51-branch, 0.0027 to 0.0067 in Decalin, 0.0016 to 0.0085 in Aroclor, with somewhat narrower ranges in the other good solvents.

\section{Method}

The storage and loss shear moduli, $G^{\prime}$ and $G^{\prime \prime}$, of the solutions were measured with the Birnboim-Schrag multiple-lumped resonator (MLR) with computerized data acquisition and processing system. ${ }^{10,11}$ Only one resonator was used, with 5 working resonances from 100 to $6000 \mathrm{~Hz}$. However, by use of solvents with different viscosities and, for the 8-branch and 24branch polymers, different temperatures, additional effective frequencies and a wider range were achieved.

\section{RESULTS}

The quantities $\left(G^{\prime} / c\right)^{\frac{1}{2}}$ and $\left(G^{\prime \prime}-\omega \eta_{\mathrm{s}}\right) / c$ were plotted against $c$ and extrapolated to zero $c$ at each frequency as described in previous studies ${ }^{2,12}$ to give the corresponding intrinsic quantities $\left[G^{\prime}\right]$ and $\left[G^{\prime \prime}\right]$. The initial concentration dependences may be described by the ratios $\gamma^{\prime}$ and $\gamma^{\prime \prime}$ which are, respectively, the limiting value at low concentrations of the ratio $\left(G^{\prime} / c\right)^{-1} \mathrm{~d}\left(G^{\prime} / c\right) / \mathrm{d} c$ and the corresponding expression with $G^{\prime \prime}-\omega \eta_{\mathrm{s}}$ substituted for $G^{\prime}$. These ratios decreased monotonically with increasing frequency as usually observed. ${ }^{2,3,12}$ At low frequencies, the ratio $\gamma=\gamma^{\prime} / \gamma^{\prime \prime}$ appeared to approach about 2 for the samples with $f=8$ and 24 , and about 4 for $f=$ 51 , but it was not certain whether low-frequency limiting values had been attained and no attempt is made to interpret them except that their magnitude is reasonable.

The extrapolated values $\left[G^{\prime}\right]$ and $\left[G^{\prime \prime}\right]$ were reduced to $\left[G^{\prime}\right]_{\mathrm{R}}=\left[G^{\prime}\right] M / R T$ and $\left[G^{\prime \prime}\right]_{\mathrm{R}}=\left[G^{\prime \prime}\right] M / R T$ and then plotted logarithmically against $\omega \eta_{s}[\eta] M / R T$ in Figures 1 and 2. This abscissa has proved to be the most useful for comparing experimental results with theory; with this plot, $\left[G^{\prime \prime}\right]_{\mathrm{R}}$ at low frequencies is represented by identical ordinate and abscissa. The observed coincidence of experimental data with this line is equivalent to confirmation of the same intrinsic 


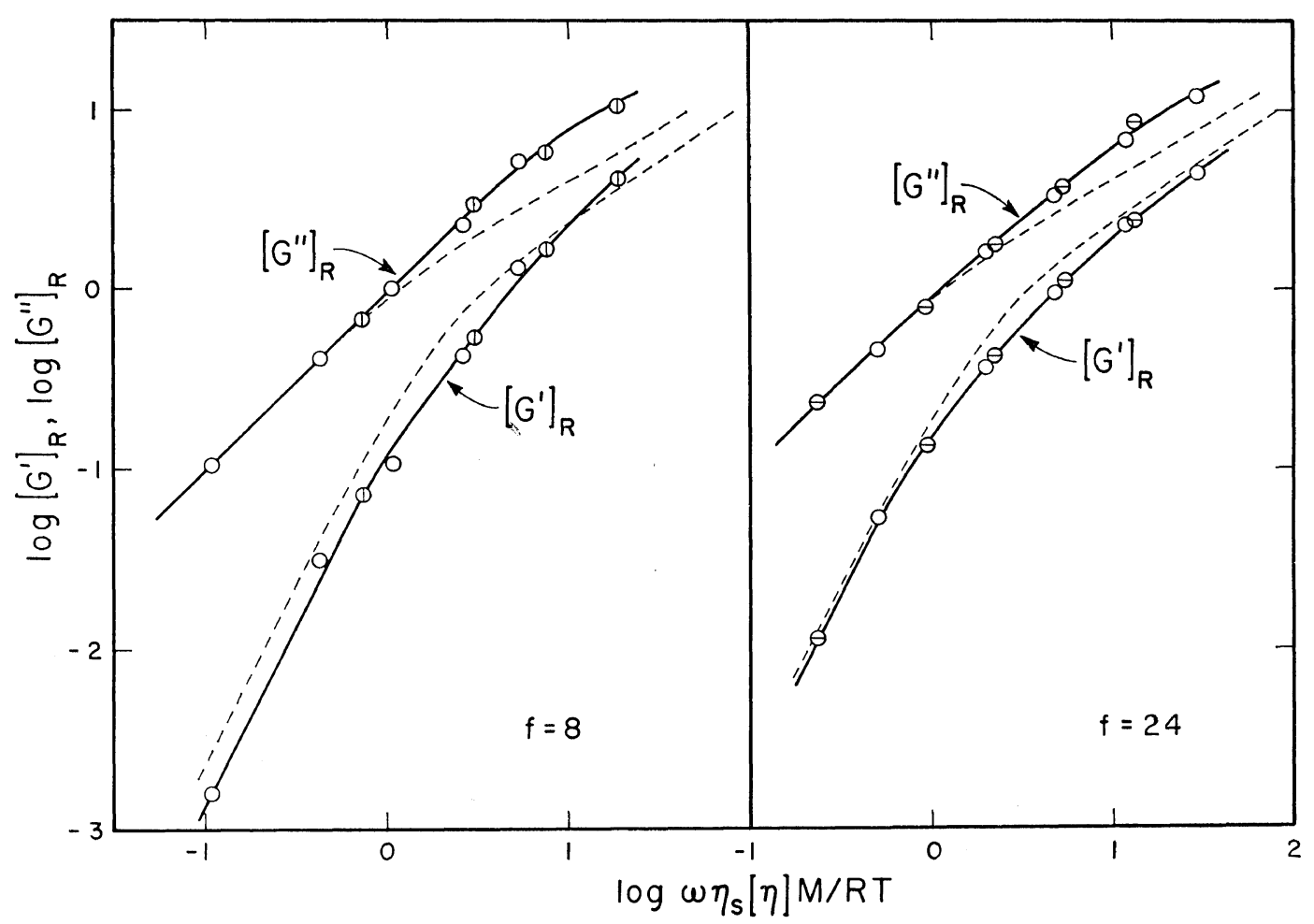

Figure 1. Logarithmic plots of reduced intrinsic shear moduli $\left[G^{\prime}\right]_{\mathrm{R}}$ and $\left[G^{\prime \prime}\right]_{\mathrm{R}}$ against $\omega \eta_{\mathrm{s}}[\eta] M / R T$ for samples with 8 and 24 branches in Aroclor 1232. Temperatures of measurement: (1), $-6.00^{\circ} \mathrm{C}$; $\bigcirc, 20.00^{\circ} \mathrm{C}$; $\ominus, 37.78^{\circ} \mathrm{C}$. Dashed lines are Zimm theory (Lodge-Wu evaluation) for a linear polymer with $h^{*}=0.15$.

viscosity from capillary and MLR measurements.

For the samples with $f=8$ and $f=24$, data at different temperatures combine to give single curves, confirming the usual conclusion that all the relevant relaxation times are proportional to solvent viscosity. For the sample with $f=51$ in three different good solvents, also, the data superposition shows that the relaxation times are proportional ${ }^{12}$ to $\eta_{\delta}[\eta]$ over a wide range of $\eta_{\mathrm{s}}$. (The $[\eta] \mathrm{s}$ are sufficiently similar so that no significant effect from differences in hydrodynamic interaction would be expected.)

In each plot, the experimental data are compared with the Zimm theory ${ }^{13}$ for a linear polymer as evaluated by Lodge and $\mathrm{Wu}^{14}$ with an appropriate value of the hydrodynamic interaction parameter $h^{*}(0.25$ for the $\Theta$-solvent, left panel of Figure 2; 0.15 for the others). These may also be regarded as experimental comparisons of branched vs. linear, since the theory correctly describes the behavior of linear polymers in this frequency range. ${ }^{1,6,12}$ At higher frequencies (above 0 on the abscissa scale), marked differences are apparent with higher slopes for the comb polymers. At the lowest frequencies, $\left[G^{\prime \prime}\right]_{R}$ must coincide for all because of the method of plotting; $\left[G^{\prime}\right]_{\mathrm{R}}$ shows some difference in the presence of greater curvature for branched polymers for $\left[G^{\prime}\right]_{R}<1$ (zero on ordinate scale).

However, it is remarkable that the magnitudes of $\left[G^{\prime}\right]_{R}$ at low frequencies are so close to those for a linear polymer and also so close to each other as shown in Figure 3. At low frequencies, the slope of $\left[G^{\prime}\right]_{R}$ on this logarithmic plot is 2 and its intercept on the ordinate axis is $\log j_{\mathrm{eR}}^{0}$, where $j_{\mathrm{eR}}^{0}$ is the intrinsic reduced steady-state compliance. In the Zimm and Zimm-Kilb theories, $j_{\mathrm{eR}}^{0}=S_{2} / S_{1}{ }^{2}$. For linear and comb polymers, $S_{1}=\Sigma\left(\tau_{p} / \tau_{1}\right)$ and $S_{2}=\Sigma\left(\tau_{p} / \tau_{1}\right)^{2}, \tau_{p}$ being 


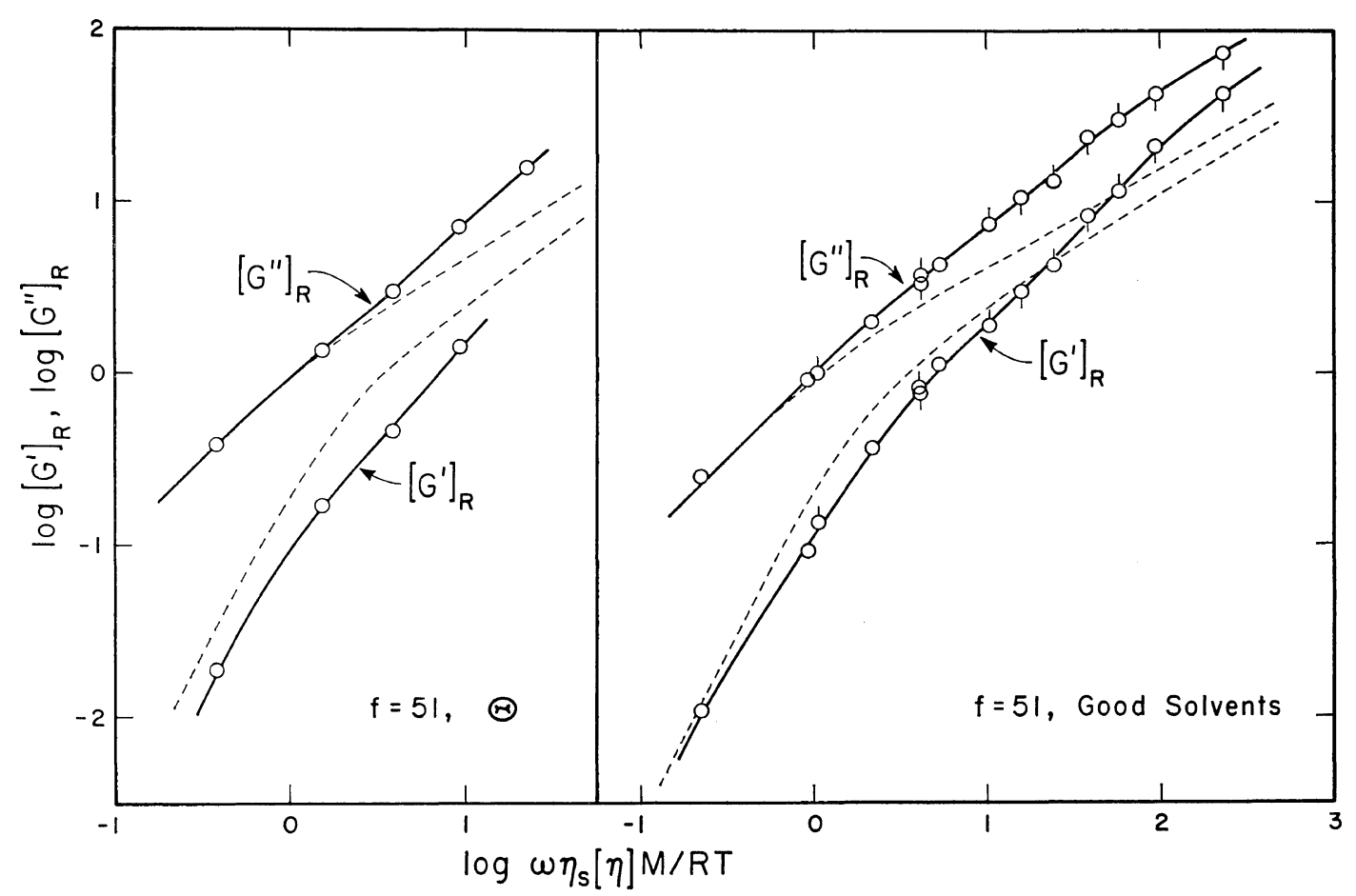

Figure 2. Logarithmic plots of reduced intrinsic shear moduli against $\omega \eta_{\mathrm{s}}[\eta] M / R T$ for sample with 51 branches, in Decalin at $15.0^{\circ} \mathrm{C}$, the $\Theta$-point (left) and in three good solvents (right): no tag, toluene, $25.0^{\circ} \mathrm{C}$; tag up, $\alpha$-chloronaphthalene, $25.0^{\circ} \mathrm{C}$; tag down, Aroclor $1232,20.0^{\circ} \mathrm{C}$. Dashed lines are Zimm theory (Lodge-Wu evaluation) for a linear polymer with $h^{*}=0.25$ (left), 0.15 (right).

the individual relaxation times and $\tau_{1}$ the longest one; for star polymer with $f$ arms,

$$
S_{i}=(f-1) \sum_{p \text { odd }}\left(\tau_{p} / \tau_{1}\right)^{i}+\sum_{p \text { even }}\left(\tau_{p} / \tau_{1}\right)^{i} .
$$

With increasing branching, $j_{\mathrm{eR}}^{0}$ is predicted to decrease rapidly, and this effect has been observed for star polymers with 4 and 9 arms. ${ }^{2,3}$ For the highly branched polymers studied here, $j_{\mathrm{eR}}^{0}$ scarcely depends on the branching, as shown in Table III. The other entries in this table will be described in the discussion.

Table III. Viscoelastic parameters

\begin{tabular}{lccccc}
\hline \multicolumn{1}{c}{$\mathrm{f}$} & $0^{\mathrm{a}}$ & 8 & 24 & 51, good & $51, \Theta$ \\
\hline $\log j_{\mathrm{eR}}^{0}$ exptl & -0.60 & -0.90 & -0.75 & -0.75 & -0.95 \\
$\log S_{2} / S_{1}^{2}$ comb theory b & & -1.13 & & & \\
$\log S_{2} / S_{1}{ }^{2}$ star theoryc $^{\mathrm{c}}$ & & -1.25 & -1.75 & -2.06 & \\
$\tau_{1, \mathrm{~b}} / \tau_{1,1}$ exptld $^{\mathrm{d}}$ & 1 & 0.16 & & 0.12 &
\end{tabular}

a For $h^{*}=0.15$.

b Osaki model with $N_{\mathrm{b}}=8 ; N_{\mathrm{s}}=1 ; h^{*}=0.25$.

c For $h^{*}=0.25$.

a With $S_{1, \mathrm{~b}}$ estimated as explained in text. 


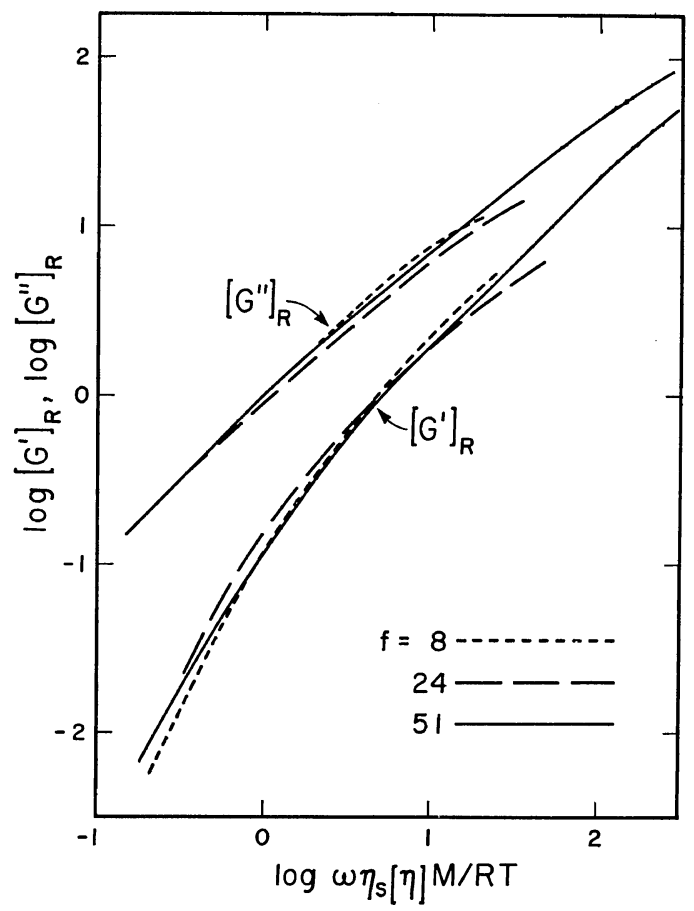

Figure 3. Experimental plots of reduced intrinsic shear moduli for three samples with indicated numbers of branches, in good solvents.

\section{DISCUSSION}

\section{Comparison with Star and Comb Theories}

The Zimm-Kilb theory has been evaluated with exact eigenvalues for star polymers with arms of equal length by Osaki and Schrag, ${ }^{5}$ and good agreement with experimental data for polymers with this geometry ${ }^{2,3}$ was obtained with reasonable assignments of $h^{*}$. (The predicted values of $\left[G^{\prime}\right]_{R}$ and $\left[G^{\prime \prime}\right]_{R}$ are not too sensitive to $h^{*}$, but there is some evidence that $h^{*}$ is inversely proportional to $\alpha_{\eta}$, the expansion factor from intrinsic viscosity.) An exact eigenvalue calculation has also been made by Osaki ${ }^{15}$ for comb polymers of specific geometry in which each branch has the same number of submolecules $\left(N_{\mathrm{b}}\right)$ and the branches are equally spaced along the backbone from each other and from the backbone ends by the same number of submolecules $\left(N_{\mathrm{s}}\right)$. The results depend on the number of branches $(f)$ and the fraction of mass in the backbone $(\lambda)$. Because of computer

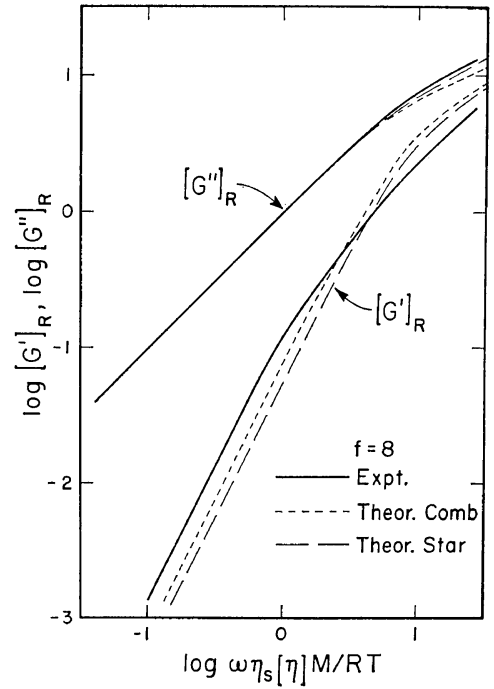

Figure 4. Experimental plot of reduced intrinsic shear moduli for sample with 8 branches compared with Zimm-Kilb theory as evaluated by Osaki and Schrag for 8-arm star and by Osaki for 8branch comb with $N_{\mathrm{b}}=8$ and $N_{\mathrm{s}}=1$ (see text); $h^{*}=0.25$.

limitations, the numbers $N_{\mathrm{b}}$ and $N_{\mathrm{s}}$ must be chosen rather unrealistically small; for $f=8$, a model with $N_{\mathrm{b}}=8$ and $N_{\mathrm{s}}=1$ gives same value of $\lambda(=0.11)$ as that of the polymer sample KI-7 with 8 branches, and this was chosen for comparison with the experimental data.

In Figure 4, the calculations for these star and comb models are compared with the experimental curves for $f=8$. Here, $h^{*}$ is taken as 0.25 because experience has indicated that branching enhances the apparent hydrodynamic interaction, ${ }^{3}$ but the choice is somewhat arbitrary and does not strongly influence the results. The two models are not very different, but the observed $\left[G^{\prime}\right]_{R}$ is higher at low frequencies and lower at higher frequencies than for either theory. The theoretical values for $\log S_{2} / S_{1}{ }^{2}$ are included in Table III.

For $f=51$, the comb calculation is not feasible, but $\lambda$ is so small (0.014) that the molecule may be regarded as not far from star configuration. The Osaki-Schrag evaluation for a 51arm star with $h^{*}=0.25$ is compared with the experimental curves in Figure 5. The theoretical prediction for $\left[G^{\prime}\right]_{R}$ at low frequencies is much too small; it reflects the rapid decrease in 


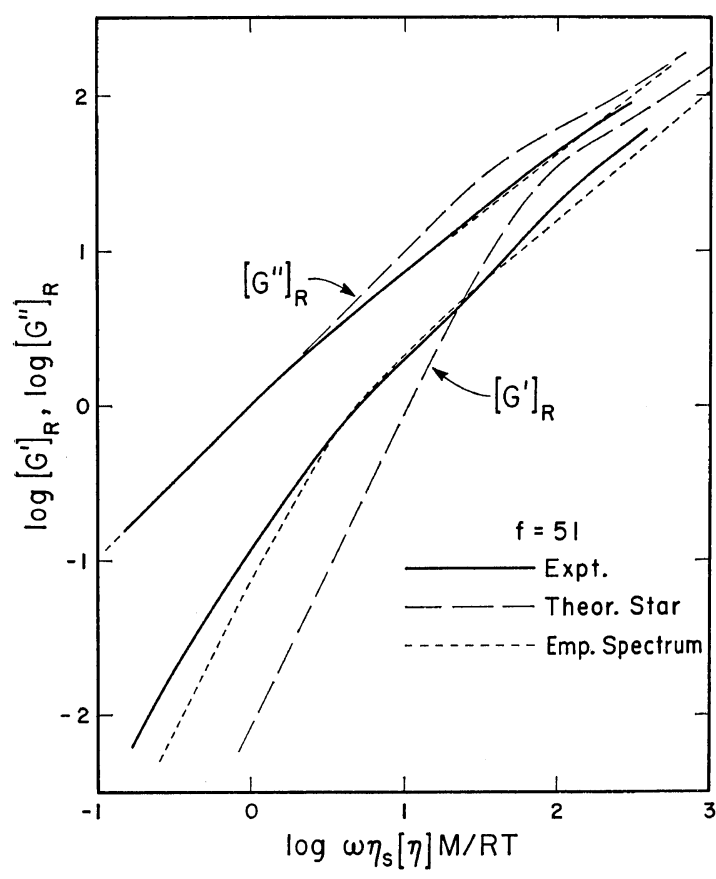

Figure 5. Experimental plot of reduced intrinsic shear moduli for sample with 51 branches compared with Zimm-Kilb theory for 51-arm comb and with empirical relaxation spectrum with $\alpha=$ 1.25 .

$S_{2} / S_{1}{ }^{2}$ with increasing $f$ expected for this model, as shown also in Table III. A different choice of $h^{*}$ would not change the comparison significantly.

Characteristic Features of Frequency Dependence

Theoretical interpretation of the results must take into account the following features.

(1) The insensitivity of $j_{\mathrm{eR}}^{0}$ to branching indicates a different distribution of the longest relaxation times from that predicted by the Zimm-Kilb theory. This is also indicated by the persistence of curvature in $\left[G^{\prime}\right]_{R}$ to frequencies well below the point where $\log \left[G^{\prime}\right]_{R}=0$ (in contrast to the case for linear molecules and stars for few arms); indeed, for $f=51$ the slope of 2 is scarcely reached even two decades below this magnitude of $\left[G^{\prime}\right]_{R}$, indicating a relaxation mechanism with an abnormally small contribution to modulus and long relaxation time.

(2) At higher frequencies, above $\log \left[G^{\prime}\right]_{R}=$ 0 , the slopes of $\log \left[G^{\prime}\right]_{R}$ and $\log \left[G^{\prime \prime}\right]_{R}$ in the "power law" region are higher than those specified by the Zimm and Zimm-Kilb theories for linear and star molecules, for which the maximum slope is $\frac{2}{3}$. Concomitantly, the ratio $\left[G^{\prime \prime}\right] /\left[G^{\prime}\right]$ is larger than the maximum value of $\sqrt{3}$ which is specified by the Zimm theory with $h^{*}=0.25$. Both these features are symptoms of a closer spacing of relaxation times; for example, for linear molecules, as $h^{*}$ goes from 0 to 0.25 , the dependence of $\tau_{p}$ on $p$ in the powerlaw zone changes from proportionality to $p^{-2}$ (Rouse) to a closer spacing of $p^{-\frac{3}{2}}$ (Zimm with dominant hydrodynamic interaction), and the ratio $\left[G^{\prime \prime}\right] /\left[G^{\prime}\right]$ goes from 1 to $\sqrt{3}$, while the logarithmic slope increases from $\frac{1}{2}$ to $\frac{2}{3}$.

In fact, by choosing a relaxation spectrum with $\tau_{p}$ proportional to an arbitrary power $p^{-\alpha}$ the intermediate frequency data can be matched rather well. In the power-law region, the slope and the $\left[G^{\prime \prime}\right] /\left[G^{\prime}\right]$ spacing are given respectively by

$$
\begin{aligned}
\mathrm{d} \log \left[G^{\prime}\right]_{\mathrm{R}} / \mathrm{d} \log \omega & =\mathrm{d} \log \left[G^{\prime \prime}\right]_{\mathrm{R}} / \mathrm{d} \log \omega \\
& =1 / \alpha
\end{aligned}
$$

and

$$
\left[G^{\prime \prime}\right] /\left[G^{\prime}\right]=\sin (\pi / 2 \alpha) / \sin [(\alpha+1) \pi / 2 \alpha]
$$

as pointed out by Spriggs. ${ }^{16}$

For the sample with $f=51$ in good solvents, the slope is about 0.8 and the ratio is about 3, both corresponding to $\alpha=1.25$. The corresponding functions for $\left[G^{\prime}\right]_{R}$ and $\left[G^{\prime \prime}\right]_{R}$, assuming that all spectral contributions to the modulus are identical, were calculated for this exponent on a UNIVAC 1108 computer, requiring 20000 terms for adequate convergence, and are plotted in Figure 5 for comparison with the experimental curve. Of course, the low-frequency region does not have any particular significance, but at intermediate frequencies the agreement is rather good.

\section{Terminal Relaxation Times}

It is impossible to calculate the terminal relaxation time $\tau_{1}$ (the additional subscript 0 denoting infinite dilution has been omitted) without definite knowledge of $S_{1} ; \tau_{1}=\eta_{\mathrm{s}}[\eta] M / R T S_{1}$. The terminal times of a branched molecule (subscript b) and a linear molecule (subscript 1) with the same molecular weight can be compared by the relation $\tau_{1, \mathrm{~b}} / \tau_{1,1}=[\eta]_{\mathrm{b}} S_{1,1} /[\eta]_{1} S_{1, \mathrm{~b}}$. In Table III, a rough approximation is made for $f=8$ taking $S_{1, \mathrm{~b}}$ from the comb theory (used in 
Figure 4) as 6.11, and for $f=51$ taking $S_{1, \mathrm{~b}}=$ 4.26 from the empirical relaxation spectrum. The ratios of 0.16 and 0.12 are not far from the value of about 0.08 observed for a 9-arm star. $^{3}$ By contrast, the Zimm-Kilb theory for a 51 -arm star (in a $\Theta$-solvent) gives the much smaller value of 0.0079 . It seems evident that the longest relaxation time in this densely branched star is much larger than the ZimmKilb theory predicts, a fact probably associated with the high concentration of polymer segments near the center of the molecule. Quantitative conclusions cannot be drawn, however, without definite knowledge of the spectral spacings.

Acknowledgment. This work was supported in part by grants from the Army Research Office (Durham), the National Institutes of Health, and the National Science Foundation. We are greatly indebted to Professor M. Nagasawa for the gift of the samples and to Dr. K. Osaki for discussion and advice.

\section{REFERENCES}

1. K. Osaki, Advan. Polym. Sci., in press.

2. K. Osaki, Y. Mitsuda, J. L. Schrag, and J. D. Ferry, Macromolecules, 5, 17 (1972).
3. Y. Mitsuda, K. Osaki, J. L. Schrag, and J. D. Ferry, Polymer J., 4, 24 (1973).

4. B. H. Zimm and R. W. Kilb, J. Polym. Sci., 37, 19 (1959).

5. K. Osaki and J. L. Schrag, ibid., Polym. Phys. Ed., 11, 549 (1973).

6. K. Osaki, J. L. Schrag, and J. D. Ferry, Macromolecules, 5, 144 (1972).

7. T. Fujimoto, H. Narukawa, and M. Nagasawa, ibid., 3, 57 (1970).

8. I. Noda, T. Horikawa, T. Kato, T. Fujimoto, and M. Nagasawa, ibid., 3, 795 (1970).

9. J.-G. Zilliox, Makromol. Chem., 156, 121 (1972).

10. J. L. Schrag and R. M. Johnson, Rev. Sci. Instrum., 42, 224 (1971).

11. D. J. Massa and J. L. Schrag, J. Polym. Sci., Part A-2, 10, 71 (1972).

12. R. M. Johnson, J. L. Schrag, and J. D. Ferry, Polymer J., 1, 742 (1970).

13. B. H. Zimm, J. Chem. Phys., 24, 269 (1956).

14. A. S. Lodge and Y.-J. Wu, University of Wisconsin Mathematics Research Center Technical Report 1250 (1972).

15. K. Osaki, Y. Mitsuda, J. L. Schrag, and J. D. Ferry, Trans. Soc. Rheol., to be submitted.

16. T. W. Spriggs, Chem. Eng. Sci., 20, 931 (1965). 